\title{
A novel computational paradigm for a precise determination of the hadronic contribution to $\left(g_{\mu}-2\right)$ from lattice QCD
}

\author{
Leonardo Giusti, ${ }^{a, b, *}$ Mattia Dalla Brida, ${ }^{a, b}$ Tim Harris $^{a, b}$ and Michele Pepe ${ }^{b}$ \\ ${ }^{a}$ Dipartimento di Fisica, Università di Milano-Bicocca \\ Piazza della Scienza 3, I-20126 Milano, Italy \\ ${ }^{b}$ INFN, Sezione di Milano-Bicocca \\ Piazza della Scienza 3, I-20126 Milano, Italy \\ E-mail: leonardo.giusti@mib.infn.it, mattia.dallabrida@mib.infn.it, \\ tim.harris@mib.infn.it, michele.pepe@mib.infn.it
}

The hadronic contribution to the muon anomalous magnetic moment $a_{\mu}=\left(g_{\mu}-2\right) / 2$ has to be determined at the per-mille level for the Standard Model prediction to match the expected final uncertainty of the ongoing E989 experiment. That is 3 times better than the current precision from the dispersive approach, and 5-15 times smaller than the uncertainty based on the purely theoretical determinations from lattice QCD. So far the stumbling-block is the large statistical error in the Monte Carlo evaluation of the required correlation functions which can hardly be tamed by brute force. In this talk we present our proposal to solve this problem by multi-level Monte Carlo integration, a technique which reduces the variance of correlators exponentially in the distance of the fields. We report the results of our feasibility tests for the computation of the Hadronic Vacuum Polarization on a lattice with a linear extension of $3 \mathrm{fm}$, a spacing of 0.065 $\mathrm{fm}$, and a pion mass of $270 \mathrm{MeV}$. Indeed the two-level integration makes the contribution to the statistical error from long-distances de-facto negligible by accelerating its inverse scaling with the cost of the simulation. These findings establish multi-level Monte Carlo as a solid and efficient method for a precise lattice determination of the hadronic contribution to $a_{\mu}$.

40th International Conference on High Energy physics - ICHEP2020

July 28 - August 6, 2020

Prague, Czech Republic (virtual meeting)

\footnotetext{
${ }^{*}$ Speaker
} 


\section{Introduction}

The measurement of the muon anomalous magnetic moment $a_{\mu}=11659208.9(6.3) \times 10^{-10}$ by the E821 experiment has the remarkable precision of 0.54 parts per million (ppm) [1], and the on-going E989 experiment at FNAL is expected to reach the astonishing precision of $0.14 \mathrm{ppm}$ by the end of its operation [2]. The Standard Model (SM) prediction includes contributions from fiveloop Quantum Electrodynamics, two-loop Weak interactions, the Hadronic leading-order Vacuum Polarization (HVP) and Hadronic Light-by-Light scattering (HLbL) [3]. The overall theoretical uncertainty is dominated by the hadronic part. So far, lacking precise purely theoretical computations, the hadronic contributions have been extracted (by assuming the SM) from experimental data via dispersive integrals (HVP \& HLbL) and low-energy effective models supplemented with the operator product expansion (HLbL). This leads to $a_{\mu}=11659181.0(4.3) \times 10^{-10}(0.37 \mathrm{ppm})$ [3], which deviates by $3-4$ standard deviations from the E821 result. That difference has been persisting for a decade and it may be a hint for New Physics.

State-of-the-art lattice Quantum Chromodynamics (QCD) determinations of the HVP are becoming competitive. At present, quoted uncertainties range between $0.6 \%$ to roughly $2 \%$, see Ref. [3] and references therein, corresponding to an overall error on $a_{\mu}$ which is still 5-15 times larger than the anticipated uncertainty from E989. The main bottleneck [3] for matching that precision is the large statistical error in the Monte Carlo evaluation of the required correlation functions. We have recently proposed [4] to solve this problem by a novel computational paradigm based on multi-level Monte Carlo integration in the presence of fermions [5, 6]. With respect to the standard approach, this strategy reduces the variance exponentially with the temporal distance of the fields. In this first feasibility study we focused on the HVP, but the strategy is general and it can be applied to the HLbL, the isospin-breaking and electromagnetic contributions as well.

\section{The signal-to-noise problem}

The HVP can be written as

$$
a_{\mu}^{\mathrm{HVP}}=\left(\frac{\alpha}{\pi}\right)^{2} \int_{0}^{\infty} d x_{0} K\left(x_{0}, m_{\mu}\right) G\left(x_{0}\right),
$$

where $\alpha$ is the electromagnetic coupling constant, $K\left(x_{0}, m_{\mu}\right)$ is a known function increasing quadratically at large $x_{0}, m_{\mu}$ is the muon mass, and $G\left(x_{0}\right)$ is the zero-momentum correlation function

$$
G\left(x_{0}\right)=\int d^{3} \mathbf{x}\left\langle J_{k}^{e m}(x) J_{k}^{e m}(0)\right\rangle
$$

of two electromagnetic currents $J_{k}^{e m}=i \sum_{i=1}^{N_{f}} q_{i} \bar{\psi}_{i} \gamma_{k} \psi_{i}$, for unexplained notation see Ref. [4]. Here we consider $N_{f}=3$, the 3 lighter quarks of QCD with the first 2 degenerate in mass, so that

$$
G\left(x_{0}\right)=G_{u, d}^{\mathrm{conn}}\left(x_{0}\right)+G_{s}^{\mathrm{conn}}\left(x_{0}\right)+G_{u, d, s}^{\mathrm{disc}}\left(x_{0}\right) .
$$

The light-connected Wick contraction $G_{u, d}^{\mathrm{conn}}\left(x_{0}\right)$ and the disconnected one $G_{u, d, s}^{\mathrm{disc}}\left(x_{0}\right)$ are the most problematic contributions with regard to the statistical error. In standard Monte Carlo computations, the relative error of the former at large time distances $\left|x_{0}\right|$ goes as

$$
\frac{\sigma_{G_{\mathrm{u}, \mathrm{d}}}^{\mathrm{conn}}\left(x_{0}\right)}{\left[G_{\mathrm{u}, \mathrm{d}}^{\mathrm{conn}}\left(x_{0}\right)\right]^{2}} \propto \frac{1}{n_{0}} e^{2\left(M_{\rho}-M_{\pi}\right)\left|x_{0}\right|},
$$




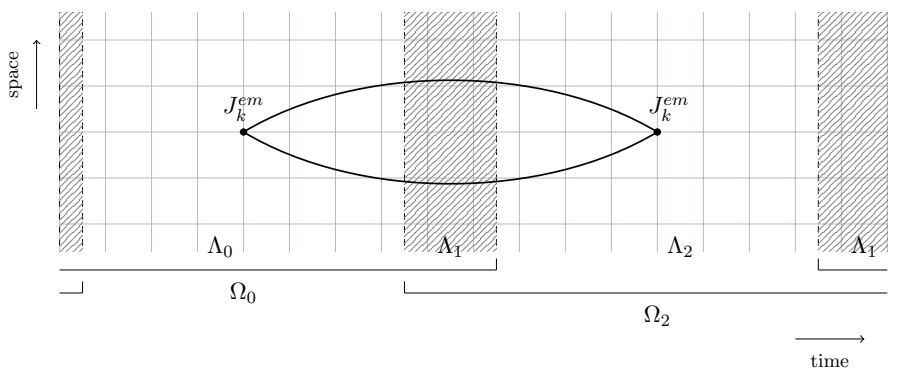

Figure 1: Domain decomposition of the lattice adopted here. Periodic (anti-periodic) boundary conditions in the time direction are enforced for gluons (fermions).

where $M_{\rho}$ is the lightest asymptotic state in the iso-triplet vector channel, and $n_{0}$ is the number of independent field configurations. Therefore the computational effort, proportional to $n_{0}$, of reaching a given relative statistical error increases exponentially with the distance $\left|x_{0}\right|$. For the disconnected contribution $G_{u, d, s}^{\mathrm{disc}}\left(x_{0}\right)$, the situation is worse since the variance is constant in time and therefore the coefficient multiplying $\left|x_{0}\right|$ is larger. At present this exponential increase of the relative error is the barrier which prevents lattice theorists to reach a per-mille statistical precision for the HVP.

\section{Multi-level Monte Carlo}

Thanks to the conceptual, algorithmic and technical progress over the last few years, it is now possible to carry out multi-level Monte Carlo simulations in the presence of fermions [5, 6]. The first step in this approach is the decomposition of the lattice in two overlapping domains $\Omega_{0}$ and $\Omega_{2}$, see e.g. Fig. 1, which share a common region $\Lambda_{1}$. The latter is chosen so that the minimum distance between the points belonging to the inner domains $\Lambda_{0}$ and $\Lambda_{2}$ remains finite and positive in the continuum limit. The next step consists in rewriting the determinant of the Hermitean massive Wilson-Dirac operator $Q=\gamma_{5} D$ as

$$
\operatorname{det} Q=\frac{\operatorname{det}(1-w)}{\operatorname{det} Q_{\Lambda_{1}} \operatorname{det} Q_{\Omega_{0}}^{-1} \operatorname{det} Q_{\Omega_{2}}^{-1}},
$$

where $Q_{\Lambda_{1}}, Q_{\Omega_{0}}$, and $Q_{\Omega_{2}}$ indicate the very same operator restricted to the domains specified by the subscript. They are obtained from $Q$ by imposing Dirichlet boundary conditions on the external boundaries of each domain. The matrix $w$ is built out of $Q_{\Omega_{0}}, Q_{\Omega_{2}}$ and the hopping terms of the operator $Q$ across the boundaries in between the inner domains $\Lambda_{0}$ and $\Lambda_{2}$ and the common region $\Lambda_{1}$ [6]. The denominator in Eq. (5) has already a factorized dependence on the gauge field since $\operatorname{det} Q_{\Lambda_{1}}$, det $Q_{\Omega_{0}}^{-1}$ and det $Q_{\Omega_{2}}^{-1}$ depend only on the gauge field in $\Lambda_{1}, \Omega_{0}$ and $\Omega_{2}$ respectively. In the last step, the numerator in Eq. (5) is rewritten as

$$
\operatorname{det}(1-w)=\frac{\operatorname{det}\left[1-R_{N+1}(1-w)\right]}{C \prod_{k=1}^{N / 2} \operatorname{det}\left[\left(u_{k}-w\right)^{\dagger}\left(u_{k}-w\right)\right]},
$$

where $u_{k}$ and $u_{k}^{*}$ are the $N$ roots of a polynomial approximant for $(1-w)^{-1}$, the numerator is the remainder, and $C$ is an irrelevant constant. The denominator in Eq. (6) can be represented by an integral over a set of $N / 2$ multi-boson fields [4-6] having an action with a factorized dependence on the gauge field in $\Lambda_{0}$ and $\Lambda_{2}$ inherited from $w$. When the polynomial approximation is properly chosen, the remainder in the numerator of Eq. (6) has mild fluctuations in the gauge field, and it is included in the observable in the form of a reweighting factor. 
A simple implementation of these ideas is to divide the lattice as shown in Fig. 1, where $\Lambda_{0}$ and $\Lambda_{2}$ have the shape of thick time-slices while $\Lambda_{1}$ includes the remaining parts of the lattice. The short-distance suppression of the quark propagator implies that a thickness of $0.5 \mathrm{fm}$ or so for the thick-time slices forming $\Lambda_{1}$ is good enough, and is not expected to vary significantly with the quark mass. This is the domain decomposition that we use for the numerical computations presented here.

The Monte Carlo simulation is then performed using a two-level scheme. We first generate $n_{0}$ level-0 gauge field configurations by updating the field over the entire lattice; then, starting from each level-0 configuration, we keep fixed the gauge field in the overlapping region $\Lambda_{1}$, and generate $n_{1}$ level- 1 configurations by updating the field in $\Lambda_{0}$ and in $\Lambda_{2}$ independently thanks to the factorization of the action. The resulting gauge fields are then combined to obtain effectively $n_{0} \cdot n_{1}^{2}$ configurations at the cost of generating $n_{0} \cdot n_{1}$ gauge fields over the entire lattice. Previous experience on twolevel integration suggests that, with two independently updated regions, the variance decreases proportionally to $1 / n_{1}^{2}$ until the standard deviation of the estimator is comparable with the signal, i.e. until the level-1 integration has solved the signal-to-noise problem. From Eq. (4) we thus infer that the variance reduction due to level-1 integration is expected to grow exponentially with the time-distance of the currents in Eq. (2).

\section{Lattice computation}

In order to assess the efficiency of two-level Monte Carlo integration, we simulated QCD with two dynamical flavours supplemented by a valence strange quark on a lattice of size $96 \times 48^{3}$ with a spacing of $a=0.065 \mathrm{fm}$, and with a pion mass of $270 \mathrm{MeV}$. The domains $\Lambda_{0}$ and $\Lambda_{2}$ are the union of 40 consecutive time-slices, while each thick time-slice forming the overlapping region $\Lambda_{1}$ is made of 8 time-slices. The determinants in the denominator of Eq. (5) are taken into account by standard pseudofermion representations, while the number of multi-bosons is fixed to $N=12$. The very same action and set of auxiliary fields are used either at level- 0 or at level- 1 . The reweighting factor is estimated stochastically with 2 random sources, which are enough for its contribution to the statistical error to be negligible. We generate $n_{0}=25$ level-0 configurations, and for each of them, we generate $n_{1}=10$ configurations in $\Lambda_{0}$ and $\Lambda_{2}$. Further details on the algorithm and its implementation can be found in Refs. [4-6]. To single out the reduction of the variance due only to two-level averaging, we carry out a dedicated calculation of correlation functions. We compute the light-connected contraction by averaging over 216 local sources put on the time-slice $y_{0} / a=32$ of $\Lambda_{0}$ which is at a distance of 8 lattice spacings from its right boundary and, as usual, by summing over the sink space-position. We determine the disconnected contraction by averaging each single-propagator trace over a large number of Gaussian random sources, namely 768, so to have a negligible random-noise contribution to the variance [4, 7].

The variance of the light-connected contribution as a function of the distance from the source is shown on the left plot of Fig. 2. For better readability only the time-slices belonging to $\Omega_{2}$ are shown, i.e. those relevant for studying the effect of two-level integration given the source position. Data are normalized to the variance obtained with the same number of sources on CLS configurations which were generated with a conventional one-level HMC. The exponential reduction of the variance with the distance from the source is manifest in the data, with the maximum gain reached from $2.5 \mathrm{fm}$ onward for $n_{1}=10$. The loss of about a factor between 2 and 3 with respect to the best possible scaling, namely $n_{1}^{2}$, either for $n_{1}=3$ or 10 (dashed lines) is compatible with the presence of a 

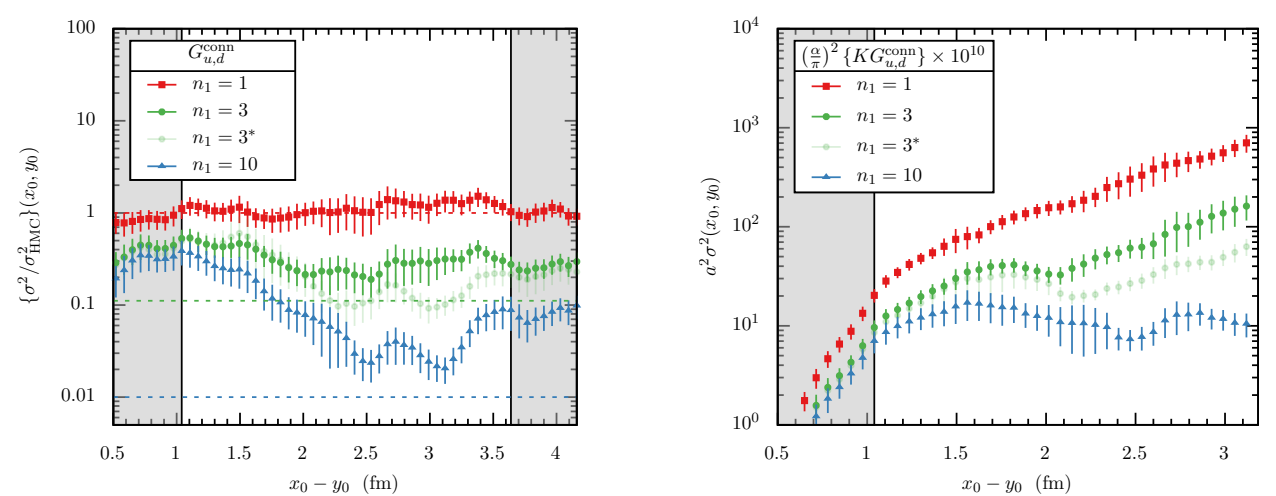

Figure 2: Left: variance of the light-connected contraction as a function of the difference between the timecoordinates of the currents for $n_{1}=1,3,10$. Data are normalized to the analogous ones computed on CLS configurations generated by one-level HMC. Dashed lines represent the maximum reduction which can be obtained by two-level integration, namely $1 / n_{1}^{2}$, in the absence of correlations between level- 1 configurations. Grey bands indicate the thick time-slices where the gauge field is kept fixed during level-1 updates. Right: variance of the light-connected contribution to the integrand in Eq. (1).

residual correlation among level-1 configurations. The power of the two-level integration can be better appreciated from the right plot of Fig. 2, where we show the variance of the light-connected contribution to the integrand in Eq. (1) as a function of the time-distance of the currents. The sharp rising of the variance computed by one-level Monte Carlo ( $n_{1}=1$, red squares) is automatically flattened out by the two-level multi-boson domain-decomposed HMC $\left(n_{1}=10\right.$, blue triangles) without the need for modeling the long-distance behaviour of $G_{u, d}^{\mathrm{conn}}\left(x_{0}\right)$.

To further appreciate the effect of the two-level integration, we compute the integral in Eq. (1) as a function of the upper extrema of integration $x_{0}^{\max }$ which we allow to vary. For $n_{1}=1$, the integral reads 446(26) and 424(38) for $x_{0}^{\max }=2.5$ and $3.0 \mathrm{fm}$ respectively, while for $n_{1}=10$ the analogous values are 467.0(8.4) and 473.4(8.6). While with the one-level integration the errors on the contributions to the integral from 0 to $2.5 \mathrm{fm}$ and from 2.5 to the maximum value of $3.0 \mathrm{fm}$ are comparable, with the two-level HMC the contribution to the variance from the long distance part becomes negligible. Considerations analogous to those made for the connected contribution apply also to the much smaller disconnected one.

\section{Results and discussion}

Our best result for the light-connected contribution to the integrand in Eq. (1) is shown on the left plot of Fig. 3 (red squares). It is obtained by a weighted average of the above discussed correlation function computed on 32 point sources per time-slice on 7 time-slices at $y_{0} / a=$ $\{8,16,24,56,64,72,80\}$ and on 216 sources at $y_{0} / a=32$. We obtain a good statistical signal up to the maximum distance of $3 \mathrm{fm}$ or so. The strange-connected contraction $G_{s}^{\text {conn }}\left(x_{0}\right)$ is much less noisy, and it is determined by averaging on 16 point sources at $y_{0} / a=32$. Its value, shown on the left plot of Fig. 3 (blue circles), is at most one order of magnitude smaller than the light contribution, and it has a negligible statistical error with respect to the light one. The best result for the disconnected contribution has been computed as discussed in the previous section, and it is shown in the left plot of Fig. 3 as well (green triangles). It reaches a negative peak at about $1.5 \mathrm{fm}$, and a good statistical signal is obtained up to $2.0 \mathrm{fm}$ or so. Its absolute value is more than two orders of magnitude smaller than the light-connected contribution over the entire range explored.

In the right plot of Fig. 3 we show the best values of the light-connected (red squares), 

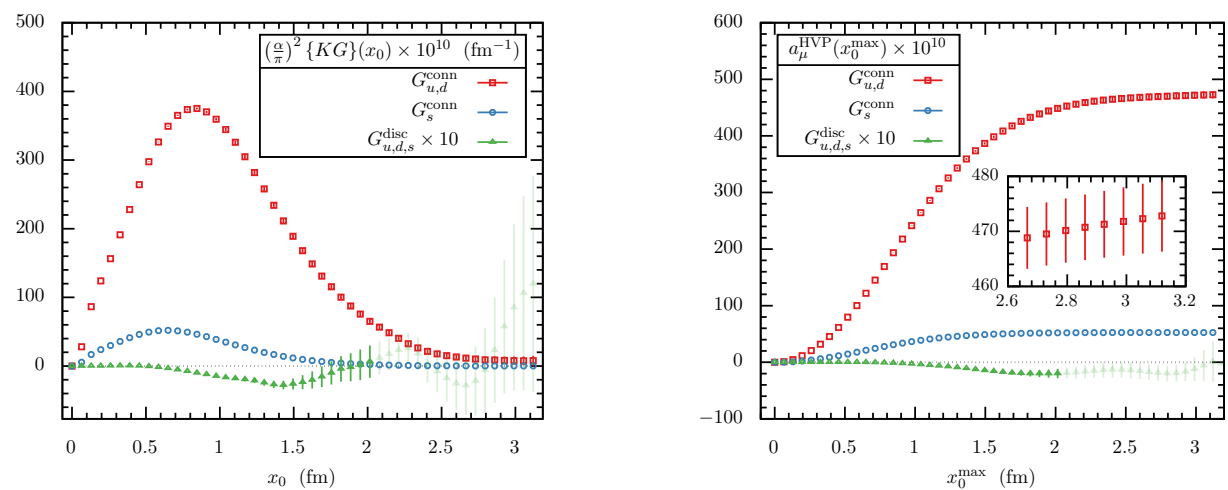

Figure 3: Left: best results for the contribution to the integrand in Eq. (1) from the light-connected (red squares), strange-connected (blue circles) and disconnected (green triangles) contractions as a function of the time coordinate. Right: best results for the contributions to $a_{\mu}^{\mathrm{HVP}}$ from light-connected (red squares), strange-connected (blue circles), and disconnected (green triangles) contractions as a function of $x_{0}^{\max }$.

strange-connected (blue circles), and disconnected (green triangles) contributions to $a_{\mu}^{\mathrm{HVP}} \cdot 10^{10}$ as a function of the upper extrema of integration $x_{0}^{\max }$ in Eq. (1). The light-connected part starts to flatten out at $x_{0}^{\max } \sim 2.5 \mathrm{fm}$ and, at the conservative distance of $x_{0}^{\max }=3.0 \mathrm{fm}$, its value is $471.8(6.2)$. The value of the strange-connected contribution is 52.55(21) at $x_{0}^{\max }=3.0 \mathrm{fm}$, and its error is negligible with respect to the light-connected one. The disconnected contribution starts to flatten out at about $x_{0}^{\max } \sim 2.0 \mathrm{fm}$, where its value is $-1.98(84)$. For $x_{0}^{\max }=3.0 \mathrm{fm}$, its statistical uncertainty is 2.1 which is still 3 times smaller with respect to the light-connected one. Clearly the disconnected contribution must be taken into account to attain a per-mille precision on the HVP, but the combined usage of split-even estimators [7] and two-level integration solves the problem of its computation. By combining the connected contributions at $x_{0}^{\max }=3.0 \mathrm{fm}$ with the disconnected part at $x_{0}^{\max }=2.0 \mathrm{fm}$, the best total value that we obtain is $a_{\mu}^{\mathrm{HVP}}=522.4(6.2) \cdot 10^{-10}$.

In this proof of concept study we have achieved a $1 \%$ statistical precision with just $n_{0} \cdot n_{1}=250$ configurations on a realistic lattice. This shows that for this light-quark mass a per-mille statistical precision on $a_{\mu}^{\mathrm{HVP}}$ is reachable with multi-level integration by increasing $n_{0}$ and $n_{1}$ by a factor of about 4-6 and 2-4 respectively. When the up and the down quarks becomes lighter, the gain due to the multi-level integration is expected to increase exponentially in the quark mass, hence improving even more dramatically the scaling of the simulation cost with respect to a standard one-level Monte Carlo. The change of computational paradigm presented here thus removes the main barrier for making affordable, on computers available today, the goal of a per-mille precision on $a_{\mu}^{\mathrm{HVP}}$.

\section{References}

[1] G. W. Bennett, et al., Phys. Rev. D73 (2006) 072003, arXiv: hep-ex/0602035.

[2] J. Grange, et al., arXiv: 1501.06858.

[3] T. Aoyama, et al., arXiv: 2006.04822.

[4] M. Dalla Brida, L. Giusti, T. Harris and M. Pepe, arXiv: 2007. 02973.

[5] M. Cè, L. Giusti, S. Schaefer, Phys. Rev. D93 (9) (2016) 094507, arXiv: 1601.04587.

[6] M. Cè, L. Giusti, S. Schaefer, Phys. Rev. D95 (3) (2017) 034503, arXiv: 1609.02419.

[7] L. Giusti, et al., Eur. Phys. J. C79 (7) (2019) 586, arXiv: 1903.10447. 